tersuchung für die Bundesebene müsste dringend um entsprechende Analysen der Koalitionen in den Ländern ergänzt werden. Jesse hält an der - aus normativer Sicht sympathischen - Forderung fest, dass die Parteien ihre Bündnisabsichten vor den Wahlen klar offenlegen sollten. Die Frage bleibt aber, ob sich das in einem Vielparteiensystem mit multiplen Koalitionsmöglichkeiten aus strategischen Gründen nicht verbietet. Hier könnte der Vergleich mit anderen europäischen Ländern Aufschluss geben, in denen man sich an den Umgang mit flexiblen Koalitionen gewöhnt hat (Benelux, Skandinavien).

Letzteres verweist auf eine gewisse Schwäche des Bandes, der die institutionellen Folgen des Parteiensystemwandels nämlich trotz seiner großen thematischen Bandbreite nur äußerst selektiv behandelt. Symptomatisch dafür sind die beiden einleitenden Beiträge von Marten Breuer und Hans Hugo Klein, die sich mit Detailproblemen des bundesdeutschen Wahlrechts befassen (getrennte Geltung der Sperrklausel für Ost- und Westdeutschland bei der ersten gesamtdeutschen Bundestagswahl 1990, Überhangmandate, Grundmandatsklausel), statt der grundsätzlichen Frage nachzugehen, ob und gegebenenfalls in welcher Form der Zersplitterung des Parteiensystems und den Oligarchisierungstendenzen der Parteiendemokratie durch einen generellen Systemwechsel begegnet werden sollte (wie dies zum Beispiel Altbundespräsident Roman Herzog unlängst gefordert hat). Der Beitrag von Hans Herbert von Arnim bleibt hier in seiner übertriebenen Selbstreferentialität ebenfalls recht unbefriedigend, auch wenn er zumindest Teilantworten liefert.

Mindestens so wichtig wie eine Reform der Abgeordnetenentschädigung und eine Stärkung der personellen Auswahlmöglichkeiten des Wählers dürften folgende durch die Pluralisierung der Parteienlandschaft aufgeworfene Fragen sein: Sind neue formelle oder informelle Regeln der Regierungsbildung erforderlich? Sollten Minderheitsregierungen zugelassen werden? Braucht es direktdemokratischen Ersatz, wenn der unmittelbare Einfluss der Wählerentscheidung auf die Regierungsbildung zurückgeht? Müssen die Parteien durch die Einführung von Urwahlen und Mitgliederentscheiden mehr Demokratie in den eigenen Reihen wagen? ${ }^{1}$ Die Bände von Niedermayer sowie Jesse und Klein haben diese Probleme noch weitgehend ausgespart; künftige Bestandsaufnahmen werden sich ihnen widmen müssen.

Frank Decker

1 Vgl. dazu Frank Decker, Veränderte Landschaft. Parteipolitik zwischen Lagerdenken und neuen Koalitionen, in: MUT. Forum für Kultur, Politik und Geschichte, Nr. 490 (Juni 2008), S. 10 - 19.

\title{
Neue Abhandlungen zur NPD: kompetenter Einstieg, analytischer Tiefgang
}

Bergsdorf, Harald: Die neue NPD. Antidemokraten im Aufwind, Olzog Verlag, München 2007, 160 Seiten, $€ 14,-$.

Backes, Uwe und Henrik Steglich (Hrsg.): Die NPD. Erfolgsbedingungen einer rechtsextremistischen Partei, Nomos Verlagsgesellschaft, Baden-Baden 2007, 426 Seiten, € 49,-.

Die sozialwissenschaftliche Debatte über den parteipolitischen Extremismus hat sich in den letzten Jahren wieder intensiviert. Insbesondere durch die Erfolge der rechtsextremen Parteien in den neuen Bundesländern ist die Aufmerksamkeit für antidemokratische Gruppie- 
rungen gestiegen. Die Politikwissenschaft trägt dem durch eine Vielzahl von aktuellen Publikationen Rechnung. Zu den neuesten Veröffentlichungen zählen die Monographie von Harald Bergsdorf und ein von Uwe Backes und Harald Steglich herausgegebener Sammelband.

$\mathrm{Zu}$ Beginn seiner Arbeit ist Bergsdorf zunächst um begriffliche Klarheit bemüht. Er spricht seinem Untersuchungsgegenstand, dem Extremismus, drei Generaleigenschaften zu. Extremisten entwickeln einen „kategorischen Wahrheitsanspruch“ (S. 18), denken in dichotomischen Freund-Feind-Kategorien (S. 19) und lehnen den demokratischen Pluralismus ab. Dies ginge mit der Priorität für das gesellschaftliche Kollektiv gegenüber dem selbstbestimmten Individuum einher. „Weil Extremisten meinen“, so Bergsdorf, „,absolute Wahrheiten bzw. Heilslehren zu besitzen, halten sie freie Wahlen für irreführend, überflüssig oder gar gefährlich“ (S. 19). Auf den ersten Blick scheinen diese Merkmale sehr allgemein und wenig trennscharf. Hier ist dem Autor jedoch zu bescheinigen, dass er mit seinem Definitionsangebot bewusst den Versuch unternimmt, den Extremismusbegriff frei von ideologischen Schlagseiten zu fassen. Lobenswert ist auch, dass der Verfasser die Unterscheidung zwischen Extremismus und Populismus herausstellt - handelt es sich bei Letztgenanntem doch um ein mit der Demokratie kompatibles, obgleich ihr langfristig schadendes, anti-elitäres Vermittlungsmuster, mit dem einige Parteien versuchen, den „gordischen Knoten moderner Politik mit dem Schwert vereinfachter Lösungen zu durchschlagen“" (S. 21).

Nachdem der Autor die historische Entwicklung der NPD nachgezeichnet hat, wendet er sich dem letzten NPD-Verbotsverfahren zu. Detailliert beschreibt er die verschiedenen Etappen und veranschaulicht so die hohen formaljuristischen Hürden eines möglichen neuen Anlaufs, die ohne eine größere Distanz zu den Informationsgebern und ihren Aussagen wohl kaum zu überwinden sind (S. 65 ff.). Hinsichtlich ihrer Programmatik bescheinigt Bergsdorf der NPD, dass sie ihre Inhalte streckenweise als reine Ideologie vorträgt, an manchen Stellen jedoch auch ganz bewusst weniger polarisierende Themen bedient - eine Tatsache, die der Verfasser mit dem Begriff des double speak zu umschreiben versucht (S. 79). Besonders interessant ist dabei das Verhältnis der NPD zur sozialen Marktwirtschaft. Bergsdorf kann zeigen, dass die Partei eine programmatische Wandlung vollzogen hat und insbesondere nach dem Amtsantritt des derzeitigen Vorsitzenden Udo Voigt einen Schwenk in die Richtung des „nationalen Sozialismus“ unternimmt (S. 94 f.). Eine antikapitalistische Ausrichtung soll, so Bergsdorf, „eine Krisenstimmung schaffen [...], um [die] Verbalattacken gegen freiheitliche Demokratie und Soziale Marktwirtschaft zu rechtfertigen“" (S. 95). Nach drei Abschnitten zu Organisation, Wählerstruktur und demoskopischem Umfeld der NPD betrachtet der Autor die medialen Auftritte von NPD-Mitgliedern und gibt abschließend Hinweise für eine politische Auseinandersetzung mit dem parteipolitischen Rechtsextremismus (12-Punkte-Programm). Hier wird deutlich, wie verkürzt und streckenweise auch wenig souverän die inhaltliche Auseinandersetzung mit der NPD insbesondere in den Medien geführt wird (S. 131 f.). Damit erfährt der extremistische Herausforderer jene Aufwertung als vorgeblich wohlmeinender politischer Märtyrer, die er bewusst anstrebt. Deswegen sei es wesentlich, sich der inhaltlichen Herausforderung anzunehmen, die von den „Halbwahrheiten " in ihren Argumentationen ausgingen (S. 136 f.). Will der demokratische Verfassungsstaat auf seine evidenten Vorzüge gegenüber totalitären Entwürfen aufmerksam machen, so setzt dies voraus, „die antidemokratische Agitation der NPD vor Ort gleichermaßen entschlossen und gelassen zu bekämpfen - ohne Hysterie und Ignoranz, ohne Über- und Untertreibungen“" (S. 141). 
Harald Bergsdorf bietet mit seinem Buch einen allgemeinen Einstieg in die aktuelle Debatte zur NPD-Problematik. Besonders die unaufgeregte und analytische Art der Betrachtung weiß zu überzeugen. Zu bemängeln ist allerdings, dass die ausgewählten Inhalte nur unzureichend begründet werden: Er bringt sie nicht mit einem ganzheitlichen inhaltlichen Erkenntnisinteresse seiner Monographie in Verbindung. So entsteht der Eindruck, dass der Versuch eines kurzen, aber thematisch möglichst allumfassenden Rundumschlags unternommen wurde. Dies freilich ist im Rahmen des veröffentlichten Umfangs schlechterdings nicht möglich. Sieht man davon ab, bietet das Buch nicht nur dem akademischen Publikum eine kompakte Einstiegsanalyse und wird damit seinem eigenen Anspruch zweifellos gerecht (S. 14).

Der Sammelband von Uwe Backes und Henrik Steglich, der auf einer Tagung des Dresdener Hannah-Arendt-Instituts beruht, greift das Problemfeld NPD deutlich umfassender auf. Den vier thematischen Einheiten des Buches - Rahmenbedingungen, Strategien, Umfeld sowie Ideologie - werden jeweils vier bis fünf Aufsätze zugewiesen. Die Fragestellungen der einzelnen Beiträge sind ausnahmslos durch ein sehr spezifisches Erkenntnisinteresse gekennzeichnet.

Patrick Moreau wirft angesichts sehr unterschiedlicher Erfolge der extremen Rechten in Europa zu Beginn des Buches die Frage auf, ob die Existenz dieser Parteiengruppierung wirklich „neue Konfliktlinien und soziale Spannungen in allen europäischen Gesellschaften [reflektiert]“ (S. 33). Der anhaltende Erfolg des rechtsextremen Lagers setzt aus seiner Sicht nicht allein „Krisen und Brüche auf politischer, wirtschaftlicher und sozialer Ebene [voraus]“. Hinzutreten müsse auch die „Dominanz einer Partei im rechtsextremen Lager“ (S. 44). Einen solchen Status strebt die NPD mit dem Deutschlandpakt zweifellos an. Neben dieser eher theoretischen Einleitung besticht der Abschnitt „Rahmenbedingungen“ vor allem durch seine empirischen Einzelanalysen. Henrik Steglich zeigt, dass der Erfolg einer Rechtspartei, über die bei Moreau genannten Makrofaktoren hinaus, auch „als Ergebnis einer komplexen, durch sogenannte Gelegenheitsstrukturen moderierten Interaktion zwischen Angebots- und Nachfrageseite [zu verstehen sei]“ (S. 56). Die empirische Analyse belegt ihm zufolge die These, dass vor allem die Themenagenda den Wahlerfolg einer rechtsextremen Partei determiniert. Diese benötigten also dringend die Nachfrage „ihrer Kernkompetenzen (Ausländer/Kriminalität)“ sowie eine „starke Anti-Establishment-Stimmung “ in der Bevölkerung. Im Erfolgsfall habe die extreme Rechte diese Stimmungen erkannt und dann auf das richtige thematische Pferd gesetzt (S. 67). Dass beide Faktoren den Erfolg begünstigen, erscheint hoch plausibel. Umso interessanter ist es, mit Steglich zu fragen, ob die Existenz solcher Stimmungen und eine entsprechende programmatische Ausrichtung von Republikanern, DVU und NPD bereits mehr ist als eine notwendige Bedingung. Angesichts ihres anhaltenden Misserfolgs bei Bundestagswahlen, trotz unverkennbarer Vertrauenseinbußen der etablierten Parteien auch auf nationaler Ebene, darf das zumindest bezweifelt werden (S. 68). Steglich rät daher davon ab, rechtsextreme Wahlerfolge monokausal zu deuten (ebenda). Tim Spier (S. 75 - 102) beschreibt daneben die regionalen Unterschiede der NPD-Erfolge in Sachsen mit Hilfe einer aufwendigen wahlökologischen Aggregatdatenanalyse. Sven Schönfelder zeigt (S. 103 - 118), dass autoritäre und fremdenfeindliche Aussagen in den neuen Bundesländern noch immer höhere Zustimmungsraten erfahren, während in den alten Ländern antisemitische Einstellungen stärker verhaftet scheinen (S. 107). Auf der Basis seiner untersuchten Aussagen entwickelt Schönfelder eine Skala zur Ermittlung rechtspopulistischen Wählerpotentials in Deutschland, das 
er für Gesamtdeutschland bei rund 19,4 und für Ostdeutschland bei 24,5 Prozent veranschlagt (S. 108 f.).

Im zweiten Abschnitt stehen die „Strategien“ der NPD im Vordergrund. Zuerst stellt Lazaros Miliopoulos die strategischen Anknüpfungspunkte der Partei im Spannungsverhältnis zwischen Programmangebot und der durch vorherrschende Gelegenheitsstrukturen gekennzeichneten Wählernachfrage dar (S. 121). Strategisches Handeln wird unter Rückgriff auf eine Kategorisierung von Joachim Raschke streng von Taktik unterschieden und als ein allgemeiner, also nicht nur situationsgebundener Ansatz zur Durchsetzung eigener Interessen verstanden. Eine politische Außenseiterposition, wie man sie der NPD aufgrund ihrer Ideologie und ihrer ideengeschichtlichen Kontinuität wird bescheinigen können, macht ein strategisches Handeln umso erforderlicher (ebenda). Der Autor arbeitet heraus, dass sich die NPD dieser Herausforderung mithilfe eines strategischen Mehrebenenkonzepts anzunehmen versucht. Miliopoulos erläutert unter anderem den von der NPD selbst proklamierten Dreiklang aus dem Kampf um die Straße (Bewegungsstrategie), die Köpfe (Strategie ideologischer Flexibilität) und die Parlamente (Professionalisierungsstrategie) (S. 123 - 135). Letzterem wenden sich drei Falluntersuchungen zur parlamentarischen Arbeit der NPD zu. Franziska Brech widmet sich der Präsenz der NPD-Fraktion im Landtag von Sachsen, die durch zwei Phasen gekennzeichnet sei: In der ersten Phase, dem Zeitraum unmittelbar nach dem Einzug in den Landtag, gaben die Abgeordneten dieser Partei „zur anfänglichen Überraschung vieler Polit-Akteure ein geordnetes Biedermann-Bild ab“ (S. 172). Befördert durch ein überdurchschnittliches Medieninteresse erarbeitete sich die Partei die gewünschte Aufmerksamkeit. Die Autorin konzediert der NPD einen „professionellen Start“, ohne hier allerdings genauer auf ihren Professionalitätsbegriff einzugehen (ebenda). Die zweite Phase, die mit der Verabschiedung der gemeinsamen Erklärung aller sächsischen Landtagsfraktionen zum Umgang mit der NPD (im Januar 2005) beginnt, sei hingegen durch das Ankommen der Partei im parlamentarischen Alltag geprägt, in dem es der Fraktion zunehmend seltener gelang, den „Spagat zwischen rechtsextremen Ansichten und dem angestrebten Saubermann-Image“ (S. 173) hinzulegen. Weiterhin wird das Auftreten der NPD in den Kommunalvertretungen Sachsens und Mecklenburg-Vorpommerns untersucht. Anhand vier ausgewählter kommunaler Vertretungskörperschaften (Stadtvertretung Anklam, Kreistage von Ludwigslust und von Ostvorpommern sowie der Bürgerschaft von Stralsund) arbeiten Hubertus Buchstein und Benjamin Fischer die Merkmale der kommunalpolitischen Aktivitäten der NPD heraus. Diese seien überraschenderweise kaum von unflätigem oder abweichendem Verhalten geprägt gewesen. Vielmehr beschrieben die Mitglieder der Kommunalverwaltungen die Amtsträger der NPD als „ruhig, freundlich und höflich“. Dies bedeutete auf der anderen Seite allerdings auch, dass diese sich deutlich aus der Alltagsarbeit in den untersuchten Gremien heraushielten.

Abschnitt 3 behandelt das Umfeld der NPD. Während Martin Thein und Dierk Borstel die umfangreiche organisatorische Vorfeldszene der NPD vorstellen, erörtert Eckhard Jesse die zukünftigen Möglichkeiten einer Auseinandersetzung mit der NPD. Er greift dabei das jüngste Verbotsverfahren auf und zeigt, dass vergangene Anträge aus seiner Sicht nicht nur nicht zum Ziel geführt haben, sondern die Demokratie vielmehr sogar „in mehrfacher Hinsicht [...] beschädigt[en]“ (S. 288). Übereilt eingeleitete Verbotsverfahren scheinen ihm demnach ebenso fragwürdig wie die Verankerung von Verfassungsklauseln, um unerwünschten Meinungsmustern mit dem generellen Beschneiden der auch ihnen zustehenden Meinungsfreiheit zu begegnen (S. 288 f.). Auch Geschäftsordnungstricks seien kein geeig- 
neter Weg (S. 293). Jesse empfiehlt daher wie auch Bergsdorf die inhaltliche Auseinandersetzung mit den Zielsetzungen der NPD. Nur so „erfährt der Bürger, was an den Parolen aus der Sicht demokratischer Kräfte nicht stimmt" (S. 294). Verbote rechtsextremistischer Vereine oder Parteien aber, so auch der Tenor im Beitrag von Julia Gerlach, haben den Fortbestand rechtsextremer Geisteshaltungen mittelfristig nicht verhindert. Im Gegenteil: „Was kurz nach der Vereinigung von kleinen Splittergruppen, Politsekten propagiert wird, verkündet heute eine bundesweit aktive Partei unter der besonderen Gunst des Grundgesetzes." (S. 258 f.)

Abschließend geht es im Sammelband um die Ideologie der NPD. Uwe Backes arbeitet das Profil der Partei im europäischen Vergleich heraus; Steffen Kailitz und Marc Brandstetter vertiefen die nationalsozialistische Ideologie der NPD. Brandstetter analysiert Parteiprogramme sowie Artikel des „Politischen Lexikons der NPD“ und der Deutschen Stimme. Hier, so der Verfasser, würde sich die NPD deutlich aggressiver als in offiziellen Dokumenten äußern und gäbe so ihren wahren Charakter preis (S. 317). Brandstetter zeichnet die programmatische Entwicklung der NPD vom Gründungsmanifest von 1964 über das Wertheimer Manifest von 1970 bis zum Düsseldorfer Programm nach, deckt zahlreiche Mythen des rechtsextremen Denkens auf und zeigt, dass die NPD nicht vollständig dem neonazistischen Spektrum zugerechnet werden kann, da hierzu wichtige Ideologieelemente fehlten (S. 335) - eine Tatsache, die seiner Meinung nach jedoch nicht verbergen kann, dass „die bürgerlich-konservativen Ideologiefragmente der Gründungs- und Frühphase weitgehend von nationalsozialistischen Fragmenten abgelöst worden sind“ (S. 335). Kailitz geht einen Schritt weiter. Insbesondere in Bezug auf die Sozialpolitik sieht er deutliche Parallelen zwischen den Forderungen der NSDAP und der NPD. „Wie die historische NSDAP“, so bemerkt er, „versucht die NPD, mit einem opulenten Programm an Versorgungsleistungen die Zustimmung der Deutschen zu gewinnen“ (S. 348).

Der von Backes und Steglich herausgegebene Band besticht vor allem durch den analytischen Tiefgang und die hohe Aktualität seiner Beiträge. Der Zuschnitt der Analyse erlaubt naturgemäß die Bearbeitung eines deutlich breiteren inhaltlichen Feldes. Dies kann und will die Monographie von Bergsdorf nicht leisten. Beide Publikationen vermitteln eine Vielzahl von Informationen, zeigen ein hohes Abstraktionsniveau und versprechen dem Leser daher gerade vor dem Hintergrund der derzeitigen politischen Debatte zur NPD einen hohen Nutzen.

Sebastian Rehse

\section{Die NPD in Fragen und Antworten: hohe Aktualität}

Virchow, Fabian und Christian Dornbusch (Hrsg.): 88 Fragen und Antworten zur NPD. Weltanschauung, Strategie und Auftreten einer Rechtspartei - und was Demokraten dagegen tun können, Wochenschau Verlag, Schwalbach/Ts. 2008, 336 Seiten, € 24,80.

Die Herausgeber Fabian Virchow und Christian Dornbusch stellen 88 Fragen zur NPD, die von insgesamt 45 Autoren in zwölf Kapiteln beantwortet werden. Ziel des Bandes ist es, „den aktuellen Stand der Forschung und seriöser journalistischer Recherche in einer Form zugänglich zu machen, die auch dem nicht wissenschaftlich geschulten Publikum einen 